\title{
Fractal Transport Phenomena through the Scale Relativity Model
}

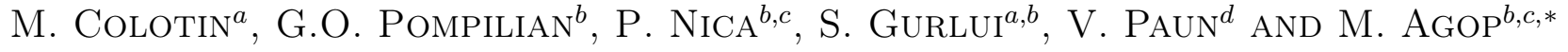 \\ ${ }^{a}$ Faculty of Physics, "Al. I. Cuza" University, Blvd. Carol I, no. 11, 700506 Iasi, Romania \\ ${ }^{b}$ Laboratoire de Physique des Lasers, Atomes et Molécules (UMR 8523), Université des Sciences et Technologies \\ de Lille, 59655 Villeneuve d'Ascq cedex, France \\ ${ }^{c}$ Department of Physics, Technical "Gh. Asachi" University \\ Blvd. Mangeron, no. 64, 700029 Iasi, Romania \\ ${ }^{d}$ Faculty of Applied Sciences, Politehnica University of Bucharest, Department of Physics I \\ 313 Splaiul Independentei Street, 060042 Bucharest, Romania
}

(Received May 21, 2009; in final form July 19, 2009)

\begin{abstract}
A correspondence between Nottale's scale relativity model and Cresson's mathematical procedures is analyzed. It results that the "synchronization" of the movements at different scales (fractal scale, differential scale etc.) gives conductive type properties to the fractal fluid, while the absence of "synchronization" is inducing properties of convective type. The behavior of a conductive fractal fluid is illustrated through the numerical simulation of plasma diffusion that is generated by laser ablation. Rotational and irrotational convective behaviors of a fractal fluid are established. Particularly, at Compton spatial and temporal scales, the irrotational behavior implies the standard Schrödinger equation.
\end{abstract}

PACS numbers: 05.45.Df, 47.53.+n, 03.65.-w

\section{Introduction}

The Nottale scale relativity (SR) model $[1-8]$ is based both on the fractal space-time concept (the fractal space-time is also introduced by Ord [9] and El Naschie [10-15]) and on a generalization of Einstein's principle of relativity to scale transformations. In other words, the SR model is built by completing the standard laws of classical physics (motion in space-time) by new scale laws ("the space-time resolution are used as intrinsic variables, playing for the scale transformation the same role as played by velocities for motion transformation" [16-19]).

Three scales of interaction of SR were developed: (i) A "Galilean" version corresponding to the standard fractals with constant fractal dimensions [20-22] and which involves quantum mechanics $[1,2,16,17,23-25]$; (ii) a special scale-relativistic version which implies the high energy physics $[2,4-6]$; (iii) a "general scale-relativistic" version which implies the cosmology $[2,3,26]$.

In the present paper the correspondence between the Nottale SR model [1-8, 16-19, 27, 28] and Cresson mathematical procedures [29-39] is analyzed. Thus, in Sect. 2 a short reminder of the Nottale SR model (mathematical

\footnotetext{
* corresponding author; e-mail: m.agop@yahoo.com
}

fundaments and physical principles) is given. Then, typical time-dependent fractal systems are considered: fractal fluid of conductive type behavior, with applications in numerical simulations of plasma expansion (Sect. 3), and fractal fluid of convective type behavior for rotational and irrotational motions (Sect. 4).

\section{A short reminder of the Nottale scale relativity theory in correspondence with Cresson's mathematical procedures}

Let us suppose that the motion of particles take place on continuous but non-differentiable curves (fractal curves). The non-differentiability, according with Cresson's mathematical procedures [29-36] and Nottale's physical principles $[1-8,16-19,27,28]$ implies the followings:

(i) a continuous and a non-differentiable curve (or almost nowhere differentiable) is explicitly scale dependent, and its length tends to infinity, when the scale interval tends to zero. In other words, a continuous and non-differentiable space is fractal, in the general meaning given by Mandelbrot to this concept [20, 21];

(ii) there is an infinity of fractals curves (geodesics) relating any couple of its points (or starting from any point), and this is valid for all scales; 
(iii) the breaking of local differential time reflection invariance. The time-derivative of a function $F$ can be written twofold

$$
\begin{gathered}
\frac{\mathrm{d} F}{\mathrm{~d} t}=\lim _{\mathrm{d} t \rightarrow 0} \frac{F(t+\mathrm{d} t)-F(t)}{\mathrm{d} t} \\
=\lim _{\mathrm{d} t \rightarrow 0} \frac{F(t)-F(t-\mathrm{d} t)}{\mathrm{d} t} .
\end{gathered}
$$

Both definitions are equivalent in the differentiable case. In the non-differentiable situation these definitions fail, since the limits are no longer defined. "In the framework of scale relativity, the physics is related to the behavior of the function during the "zoom" operation on the time resolution $\delta t$, here identified with the differential element $\mathrm{d} t$ ("substitution principle"), which is considered as an independent variable. The standard function $F(t)$ is therefore replaced by a fractal function $F(t, \mathrm{~d} t$ ) (for details see [29-36]) explicitly dependent on the time resolution interval, whose derivative is undefined only at the unobservable limit $\mathrm{d} t \rightarrow 0$ " $[16,17]$. As a consequence, this leads us to define the two derivatives of the fractal function as explicit functions of the two variables $t$ and $\mathrm{d} t$,

$$
\begin{aligned}
& \frac{\mathrm{d}_{+} F}{\mathrm{~d} t}=\lim _{\mathrm{d} t \rightarrow 0_{+}} \frac{F(t+\mathrm{d} t, \mathrm{~d} t)-F(t, \mathrm{~d} t)}{\mathrm{d} t}, \\
& \frac{\mathrm{d}_{-} F}{\mathrm{~d} t}=\lim _{\mathrm{d} t \rightarrow 0_{-}} \frac{F(t, \mathrm{~d} t)-F(t-\mathrm{d} t, \mathrm{~d} t)}{\mathrm{d} t} .
\end{aligned}
$$

The sign "+" corresponds to the forward process and "-" to the backward process;

(iv) the differential of a fractal function $F(t, \mathrm{~d} t)$ can be expressed as the sum of two differentials, one which is not scale-dependent, $\mathrm{d} F^{\prime}(t)$, and the other dependent on it, $\mathrm{d} F^{\prime \prime}(t, \mathrm{~d} t)$, therefore [29-36]

$$
\mathrm{d} F(t, \mathrm{~d} t)=\mathrm{d} F^{\prime}(t)+\mathrm{d} F^{\prime \prime}(t, \mathrm{~d} t) \text {. }
$$

Particularly, the differential of the generalized coordinates, $\mathrm{d}_{ \pm} \boldsymbol{X}(t, \mathrm{~d} t)$, can be decomposed as follows:

$$
\mathrm{d}_{ \pm} \boldsymbol{X}(t, \mathrm{~d} t)=\mathrm{d}_{ \pm} \boldsymbol{x}(t)+\mathrm{d}_{ \pm} \boldsymbol{\xi}(t, \mathrm{~d} t)
$$

where $\mathrm{d}_{ \pm} \boldsymbol{x}(t)$ is the "classical part" and $\mathrm{d}_{ \pm} \boldsymbol{\xi}(t, \mathrm{~d} t)$ is the "fractal part". Starting from here, multiplying by $\mathrm{d} t^{-1}$ and using the substitutions

$$
\boldsymbol{V}_{ \pm}=\frac{\mathrm{d}_{ \pm} \boldsymbol{X}}{\mathrm{d} t}, \quad \boldsymbol{v}_{ \pm}=\frac{\mathrm{d}_{ \pm} \boldsymbol{x}}{\mathrm{d} t}, \quad \boldsymbol{u}_{ \pm}=\frac{\mathrm{d}_{ \pm} \boldsymbol{\xi}}{\mathrm{d} t},
$$

we obtain the velocity field

$$
\boldsymbol{V}_{ \pm}=\boldsymbol{v}_{ \pm}+\boldsymbol{u}_{ \pm}
$$

(v) the fractal part of $F$, i.e. $F^{\prime \prime}$, satisfies the relation [29-32]:

$$
\left|F^{\prime \prime}(t)-F^{\prime \prime}\left(t^{\prime}\right)\right| \approx\left|t-t^{\prime}\right|^{\delta},
$$

where $\delta$ depends on the fractal dimension $D_{\mathrm{F}}$ (for detail see [30]).

Particularly, the differential of the "fractal part" of $\mathrm{d}_{ \pm} \boldsymbol{X}$, becomes

$$
\mathrm{d}_{ \pm} \xi_{i} \sim \mathrm{d} t^{\frac{1}{D_{\mathrm{F}}}}
$$

or more, as an equality relation

$$
\left(\frac{\mathrm{d}_{ \pm} \xi_{i}}{\lambda}\right)=\left(\frac{\mathrm{d} t}{\tau}\right)^{\frac{1}{D_{\mathrm{F}}}} .
$$

Written as

$$
\mathrm{d}_{ \pm} \xi_{i}=\frac{\lambda}{\tau}\left(\frac{\mathrm{d} t}{\tau}\right)^{\left(\frac{1}{D_{\mathrm{F}}}\right)-1} \mathrm{~d} t
$$

Eqs. (9a,b) imply the temporal scales $\delta t$ and $\tau$, and the length scale $\lambda$, respectively. The significances of the time $\mathrm{d} t$ and $\tau$ result from the random walk (Brownian motion) or its generalization, Levy motion $[2,10]$. The differential time $\mathrm{d} t$ is identified with the resolution time ("substitution principle" $[1-8]), \delta t \equiv \mathrm{d} t$, while $\tau$ corresponds to the fractal-non-fractal transition time. $\lambda$ is a characteristic length, for example of Planck's or de Broglie's type (for details see $[2,10])$;

(vi) by the relation $(10 \mathrm{a}, \mathrm{b})$ the velocity field $V_{ \pm}^{i}$ becomes

$$
V_{ \pm}^{i}=v_{ \pm}^{i}+u_{ \pm}^{i}=v_{ \pm}^{i}+\frac{\lambda}{\tau}\left(\frac{\tau}{\mathrm{d} t}\right)^{1-\left(\frac{1}{D_{\mathrm{F}}}\right)} .
$$

The transition scale $\tau$ yields two distinct behaviors of the speed, depending on the resolution at which it is considered, since $V_{ \pm}^{i} \rightarrow v_{ \pm}^{i}$ when $\mathrm{d} t \gg \tau$, and $V_{ \pm}^{i} \rightarrow u_{ \pm}^{i}$, when $\mathrm{d} t \ll \tau$;

(vii) the local differential time reflection invariance is recovered by combining the two derivatives, $\mathrm{d}_{+} / \mathrm{d} t$ and $\mathrm{d}_{-} / \mathrm{d} t$, in the complex operator $[2-8,29-36,27,28]$

$$
\frac{\hat{\mathrm{d}}}{\mathrm{d} t}=\frac{1}{2}\left(\frac{\mathrm{d}_{+}+\mathrm{d}_{-}}{\mathrm{d} t}\right)-\frac{\mathrm{i}}{2}\left(\frac{\mathrm{d}_{+}-\mathrm{d}_{-}}{\mathrm{d} t}\right) .
$$

We call this procedure "an extension by differentiability" (Cresson's extension - for detail see [33-36]).

Applying this operator to the "position vector" yields a complex speed

$$
\begin{aligned}
\mathcal{V} & =\frac{\hat{\mathrm{d}} \boldsymbol{X}}{\mathrm{d} t}=\frac{1}{2}\left(\frac{\mathrm{d}_{+} \boldsymbol{X}+\mathrm{d}_{-} \boldsymbol{X}}{\mathrm{d} t}\right) \\
& -\frac{\mathrm{i}}{2}\left(\frac{\mathrm{d}_{+} \boldsymbol{X}-\mathrm{d}_{-} \boldsymbol{X}}{\mathrm{d} t}\right) \\
& =\frac{\boldsymbol{V}_{+}+\boldsymbol{V}_{-}}{2}-\mathrm{i} \frac{\boldsymbol{V}_{+}-\boldsymbol{V}_{-}}{2} \\
& =\frac{1}{2}\left[\left(\boldsymbol{v}_{+}+\boldsymbol{v}_{-}\right)+\left(\boldsymbol{u}_{+}+\boldsymbol{u}_{-}\right)\right] \\
& -\frac{\mathrm{i}}{2}\left[\left(\boldsymbol{v}_{+}-\boldsymbol{v}_{-}\right)+\left(\boldsymbol{u}_{+}-\boldsymbol{u}_{-}\right)\right]=\boldsymbol{V}-\mathrm{i} \boldsymbol{U} \\
\boldsymbol{V} & =\frac{\boldsymbol{V}_{+}+\boldsymbol{V}_{-}}{2}=\frac{1}{2}\left[\left(\boldsymbol{v}_{+}+\boldsymbol{v}_{-}\right)+\left(\boldsymbol{u}_{+}+\boldsymbol{u}_{-}\right)\right], \\
\boldsymbol{U} & =\frac{\boldsymbol{V}_{+}-\boldsymbol{V}_{-}}{2}=\frac{1}{2}\left[\left(\boldsymbol{v}_{+}-\boldsymbol{v}_{-}\right)+\left(\boldsymbol{u}_{+}-\boldsymbol{u}_{-}\right)\right] .
\end{aligned}
$$

with

The real part, $\boldsymbol{V}$, of the complex speed, $\mathcal{V}$, represents the standard classical speed, which is differentiable and independent of resolution, while the imaginary part, $\boldsymbol{U}$, is a new quantity arising from fractality, which is non-differentiable and resolution-dependent. In the usual classical limit, $\mathrm{d} t \gg \tau$,

$$
\boldsymbol{v}_{+}=\boldsymbol{v}_{-}=\boldsymbol{v}, \quad \boldsymbol{u}_{+}=\boldsymbol{u}_{-}=0,
$$

so that 
$\boldsymbol{V}=\boldsymbol{v}, \quad \boldsymbol{U}=0$.

In the limit, $\mathrm{d} t \ll \tau$,

$$
\boldsymbol{v}_{+}=\boldsymbol{v}_{-}=0, \quad \boldsymbol{u}_{+}=\boldsymbol{u}_{-}=\boldsymbol{u}
$$

and

$$
\boldsymbol{V}=\boldsymbol{u}, \quad \boldsymbol{U}=0
$$

(viii) "in order to account for the infinity of geodesics in the bundle, for their fractality and for the two valuedness of the derivative which all come from the non-differentiable geometry of the space-time continuum, one therefore adopts a generalized statistical fluid like description, where instead of a classical deterministic speed or of a classical fluid speed field, one uses a doublet of fractal functions of spaces coordinates and time which are also explicit functions of resolution time" [16, 17]. Thus, the average values of the quantities must be considered in the previously mentioned sense [29-36]. Particularly, the average of $\mathrm{d}_{ \pm} \boldsymbol{X}$ is

$$
\left\langle\mathrm{d}_{ \pm} \boldsymbol{X}\right\rangle=\mathrm{d}_{ \pm} \boldsymbol{x}
$$

with

$$
\left\langle\mathrm{d}_{ \pm} \boldsymbol{\xi}\right\rangle=0
$$

(ix) in such an interpretation, the "particles" are identified with the geodesics themselves. As a consequence, any measurement is interpreted as a sorting out (or selection) of the geodesics by the measuring device [16, 17].

Let us now assume that the movement curves (continuous but non-differentiable) are immersed in a 3dimensional space, and that $\boldsymbol{X}$ of components $X^{i}(i=$ $\overline{1,3})$ is the position vector of a point on the curve. Let us also consider a function $f(\boldsymbol{X}, t)$ and the following Taylor series expansion, up to the second order

$$
\begin{gathered}
\mathrm{d}_{ \pm} f=\frac{\partial f}{\partial t} \mathrm{~d} t+\nabla f \cdot \mathrm{d}_{ \pm} \boldsymbol{X} \\
+\frac{1}{2} \frac{\partial^{2} f}{\partial X^{i} \partial X^{j}} \mathrm{~d}_{ \pm} X^{i} \mathrm{~d}_{ \pm} X^{j} .
\end{gathered}
$$

The relations $(21 \mathrm{a}, \mathrm{b})$ are valid in any point of the space-time manifold and also for the points " $\boldsymbol{X}$ " on the fractal curve which we have selected in relations $(21 \mathrm{a}, \mathrm{b})$.

From here, the forward and backward average values of this relation, take the form

$$
\begin{gathered}
\left\langle\mathrm{d}_{ \pm} f\right\rangle=\left\langle\frac{\partial f}{\partial t} \mathrm{~d} t\right\rangle+\left\langle\nabla f \cdot \mathrm{d}_{ \pm} \boldsymbol{X}\right\rangle \\
+\frac{1}{2}\left\langle\frac{\partial^{2} f}{\partial X^{i} \partial X^{j}} \mathrm{~d}_{ \pm} X^{i} \mathrm{~d}_{ \pm} X^{j}\right\rangle .
\end{gathered}
$$

We make the following stipulations: the mean values of the function $f$ and its derivates coincide with themselves and the differentials $\mathrm{d}_{ \pm} X^{i}$ and $\mathrm{d} t$ are independent. Therefore the averages of their products coincide with the product of average. Thus, Eqs. (22a,b) become

$$
\begin{aligned}
& \mathrm{d}_{ \pm} f=\frac{\partial f}{\partial t} \mathrm{~d} t+\nabla f\left\langle\mathrm{~d}_{ \pm} \boldsymbol{X}\right\rangle \\
& +\frac{1}{2} \frac{\partial^{2} f}{\partial X^{i} \partial X^{j}}\left\langle\mathrm{~d}_{ \pm} X^{i} \mathrm{~d}_{ \pm} X^{j}\right\rangle
\end{aligned}
$$

or more, using Eqs. (4a,b) with the properties (20a,b),

$$
\mathrm{d}_{ \pm} f=\frac{\partial f}{\partial t} \mathrm{~d} t+\nabla f \mathrm{~d}_{ \pm} \boldsymbol{x}
$$

$$
+\frac{1}{2} \frac{\partial^{2} f}{\partial X^{i} \partial X^{j}}\left(\mathrm{~d}_{ \pm} x^{i} \mathrm{~d}_{ \pm} x^{j}+\left\langle\mathrm{d} \xi_{ \pm}^{i} \mathrm{~d} \xi_{ \pm}^{j}\right\rangle\right) .
$$

Even the average value of the fractal coordinate, $\mathrm{d} \xi_{ \pm}^{i}$, is null (see $(20 a, b))$, for the higher order of the fractal coordinate average, the situation can be different. Let us focus on the mean $\left\langle\mathrm{d} \xi_{ \pm}^{i} \mathrm{~d} \xi_{ \pm}^{j}\right\rangle$. If $i \neq j$ this average is zero due the independence of $\mathrm{d} \xi^{i}$ and $\mathrm{d} \xi^{j}$. So, using (10a,b) we can write (see also [27, 28]):

$$
\left\langle\mathrm{d} \xi_{ \pm}^{i} \mathrm{~d} \xi_{ \pm}^{j}\right\rangle= \pm \delta^{i j} \frac{\lambda^{2}}{\tau}\left(\frac{\mathrm{d} t}{\tau}\right)^{\left(\frac{2}{D_{\mathrm{F}}}\right)-1}
$$

with

$$
\delta^{i j}=\left\{\begin{array}{lll}
1 & \text { if } & i=j \\
0 & \text { if } & i \neq j
\end{array}\right.
$$

and we had considered that

$$
\begin{aligned}
& \left\langle\mathrm{d} \xi_{+}^{i} \mathrm{~d} \xi_{+}^{j}\right\rangle>0 \quad \text { and } \quad \mathrm{d} t>0, \\
& \left\langle\mathrm{~d} \xi_{-}^{i} \mathrm{~d} \xi_{-}^{j}\right\rangle>0 \quad \text { and } \\
& \mathrm{d} t<0 .
\end{aligned}
$$

Then Eqs. (24a,b) may be written under the form

$$
\begin{gathered}
\mathrm{d}_{ \pm} f=\frac{\partial f}{\partial t} \mathrm{~d} t+\nabla f \mathrm{~d}_{ \pm} \boldsymbol{x}+\frac{1}{2} \frac{\partial^{2} f}{\partial X^{i} \partial X^{j}} \mathrm{~d}_{ \pm} x^{i} \mathrm{~d}_{ \pm} x^{j} \\
\pm \frac{\partial^{2} f}{\partial X^{i} \partial X^{j}} \delta^{i j} \frac{\lambda^{2}}{2 \tau}\left(\frac{\mathrm{d} t}{\tau}\right)^{\left(\frac{2}{D_{\mathrm{F}}}\right)-1} \mathrm{~d} t .
\end{gathered}
$$

If we divide by $\mathrm{d} t$, and neglect the terms which contain differential factors (for details on the method see [23]), Eqs. $(26 \mathrm{a}, \mathrm{b})$ are reduced to

$$
\frac{\mathrm{d}_{ \pm} f}{\mathrm{~d} t}=\frac{\partial f}{\partial t}+\boldsymbol{V}_{ \pm} \nabla f \pm \frac{\lambda^{2}}{2 \tau}\left(\frac{\mathrm{d} t}{\tau}\right)^{\left(\frac{2}{D_{\mathrm{F}}}\right)-1} \Delta f
$$

with $\nabla^{2}=\sum_{i} \frac{\partial^{2}}{\partial X_{i}^{2}}$. These relations also allow us to define the operator

$$
\frac{\mathrm{d}_{ \pm}}{\mathrm{d} t}=\frac{\partial}{\partial t}+\boldsymbol{V}_{ \pm} \nabla \pm \frac{\lambda^{2}}{2 \tau}\left(\frac{\mathrm{d} t}{\tau}\right)^{\left(\frac{2}{D_{\mathrm{F}}}\right)-1} \Delta
$$

- see also [27, 28]. Under these circumstances, let us calculate $\hat{\mathrm{d}} f / \mathrm{d} t$. Taking into account Eqs. (12), (13), and $(28 \mathrm{a}, \mathrm{b})$, we obtain

$$
\begin{aligned}
\frac{\hat{\mathrm{d}} f}{\mathrm{~d} t} & =\frac{1}{2}\left[\left(\frac{\mathrm{d}_{+} f}{\mathrm{~d} t}+\frac{\mathrm{d}_{-} f}{\mathrm{~d} t}\right)-\mathrm{i}\left(\frac{\mathrm{d}_{+} f}{\mathrm{~d} t}-\frac{\mathrm{d}_{-} f}{\mathrm{~d} t}\right)\right] \\
& =\frac{1}{2}\left[\left(\frac{\partial f}{\partial t}+\boldsymbol{V}_{+} \nabla f+\frac{\lambda^{2}}{2 \tau}\left(\frac{\mathrm{d} t}{\tau}\right)^{\left(\frac{2}{D_{\mathrm{F}}}\right)-1} \Delta f\right)\right. \\
& \left.+\left(\frac{\partial f}{\partial t}+\boldsymbol{V}_{-} \nabla f-\frac{\lambda^{2}}{2 \tau}\left(\frac{\mathrm{d} t}{\tau}\right)^{\left(\frac{2}{D_{\mathrm{F}}}\right)-1} \Delta f\right)\right] \\
& -\frac{i}{2}\left[\left(\frac{\partial f}{\partial t}+\boldsymbol{V}_{+} \nabla f+\frac{\lambda^{2}}{2 \tau}\left(\frac{\mathrm{d} t}{\tau}\right)^{\left(\frac{2}{D_{\mathrm{F}}}\right)-1} \Delta f\right)\right. \\
& \left.-\left(\frac{\partial f}{\partial t}+\boldsymbol{V}_{-} \nabla f-\frac{\lambda^{2}}{2 \tau}\left(\frac{\mathrm{d} t}{\tau}\right)^{\left(\frac{2}{D_{\mathrm{F}}}\right)-1} \Delta f\right)\right]
\end{aligned}
$$




$$
\begin{aligned}
& =\frac{\partial f}{\partial t}+\left(\frac{\boldsymbol{V}_{+}+\boldsymbol{V}_{-}}{2}-\mathrm{i} \frac{\boldsymbol{V}_{+}-\boldsymbol{V}_{-}}{2}\right) \nabla f \\
& -\mathrm{i} \frac{\lambda^{2}}{2 \tau}\left(\frac{\mathrm{d} t}{\tau}\right)^{\left(\frac{2}{D_{\mathrm{F}}}\right)-1} \Delta f=\frac{\partial f}{\partial t}+\mathcal{V} \cdot \nabla f \\
& -\mathrm{i} \frac{\lambda^{2}}{2 \tau}\left(\frac{\mathrm{d} t}{\tau}\right)^{\left(\frac{2}{D_{\mathrm{F}}}\right)-1} \Delta f .
\end{aligned}
$$

This relation also allows us to define the fractal operator $[27,28]$ :

$$
\frac{\hat{\mathrm{d}}}{\mathrm{d} t}=\frac{\partial}{\partial t}+\mathcal{V} \cdot \nabla-\mathrm{i} \frac{\lambda^{2}}{2 \tau}\left(\frac{\mathrm{d} t}{\tau}\right)^{\left(\frac{2}{D_{\mathrm{F}}}\right)-1} \Delta .
$$

We now apply the principle of scale covariance (for details see $[27,28])$, and postulate that the passage from classical (differentiable) mechanics to the "fractal" mechanics, which is considered here, can be implemented by replacing the standard time-derivative, $\mathrm{d} / \mathrm{d} t$, by the complex operator $(\hat{\mathrm{d}} / \mathrm{d} t)$ (this result is the principle of scale covariance given by Nottale in [1-8, 27, 28]). "This operator $(\hat{d} / d t)$ plays the role of a "covariant derivative operator", namely, it is used to write the fundamental equation of dynamics under the same form as in the classical and differentiable case.

Under its above form, the covariant derivative operator is not itself fully covariant since it involves second order derivative terms, while it is a first order time derivative. These second order terms imply that the Leibniz rule for a product is no longer the first order Leibniz rule, but a linear combination of the first and second order rules.

The strong covariance can be fully implemented by introducing new tools allowing us to keep the form of the first order Leibniz rule, despite the presence of the second order derivates $[16,17] "$. In this purpose, one defines the complex speed operator

$$
\hat{\mathcal{V}}=\mathcal{V}-\mathrm{i} \frac{\lambda^{2}}{2 \tau}\left(\frac{\mathrm{d} t}{\tau}\right)^{\left(\frac{2}{D_{\mathrm{F}}}\right)-1} \nabla
$$

— see also [16, 17, 27, 28].

Particularly, for movements on fractal curves of the Peano type, i.e. in the fractal dimension $D_{\mathrm{F}} \equiv 2$, the complex speed operator (31) takes the form given in [16, 17],

$$
\hat{\mathcal{V}}_{D_{\mathrm{F}}=2}=\mathcal{V}-\mathrm{i} \frac{\lambda^{2}}{2 \tau} \nabla
$$

Thus, the covariant derivative recovers the standard first order form of a total derivative in terms of partial derivatives, namely, the strong covariance Nottale principle $[16,17,27,28]$ :

$$
\frac{\hat{\mathrm{d}}}{\mathrm{d} t}=\frac{\partial}{\partial t}+\hat{\mathcal{V}} \cdot \nabla \text {. }
$$

\section{Fractal fluids of conductive type behavior}

As a consequence, we are now able to write the conservation law of the fractal function $\varepsilon$ in a fractal space-time under its strong covariant form

$$
\begin{aligned}
\frac{\hat{\mathrm{d}} \varepsilon}{\mathrm{d} t} & =\frac{\partial \varepsilon}{\partial t}+\hat{\mathcal{V}} \cdot \nabla \varepsilon \\
& \equiv \frac{\partial \varepsilon}{\partial t}+\mathcal{V} \cdot \nabla \varepsilon-\mathrm{i} \frac{\lambda^{2}}{2 \tau}\left(\frac{\mathrm{d} t}{\tau}\right)^{\left(\frac{2}{D_{\mathrm{F}}}\right)-1} \Delta \varepsilon=0
\end{aligned}
$$

or more, by separating the real and imaginary parts,

$$
\begin{aligned}
& \frac{\partial \varepsilon}{\partial t}+\boldsymbol{V} \cdot \nabla \varepsilon=0 \\
& -\boldsymbol{U} \cdot \nabla \varepsilon=\frac{\lambda^{2}}{\tau}\left(\frac{\mathrm{d} t}{\tau}\right)^{\left(\frac{2}{D_{\mathrm{F}}}\right)-1} \Delta \varepsilon .
\end{aligned}
$$

Consequently, at the differentiable scale the local temporal variation, $\partial \varepsilon / \partial t$, and the term, $\boldsymbol{V} \cdot \nabla \varepsilon$, are equal, while at the non-differentiable scale, the term, $\boldsymbol{U} \cdot \nabla \varepsilon$, and $\Delta \varepsilon$, compensate each other.

Particularly, for $\boldsymbol{V}=\boldsymbol{U}$ (i.e. "synchronal" movements at different scales), from $(35 \mathrm{a}, \mathrm{b})$ we get the diffusion type equation,

$$
\frac{\partial \varepsilon}{\partial t}=\frac{\lambda^{2}}{\tau}\left(\frac{\mathrm{d} t}{\tau}\right)^{\left(\frac{2}{D_{\mathrm{F}}}\right)-1} \Delta \varepsilon .
$$

Such an equation is implied by the Fourier type law

$$
\boldsymbol{j}(\varepsilon)=\frac{\lambda^{2}}{\tau}\left(\frac{\mathrm{d} t}{\tau}\right)^{\left(\frac{2}{D_{\mathrm{F}}}\right)-1} \nabla \varepsilon
$$

with a current density $\boldsymbol{j}(\varepsilon)$. Therefore, Eqs. (36) and (37) describe the fractal fluid of conductive type behavior.

Particularly, for movements on fractal curves of the Peano type, i.e. in the fractal dimension $D_{\mathrm{F}} \equiv 2$, Eqs. (36) and (37) take the standard forms

$$
\frac{\partial \varepsilon}{\partial t}=\frac{\lambda^{2}}{\tau} \Delta \varepsilon
$$

and respectively

$$
\boldsymbol{j}(\varepsilon)=\frac{\lambda^{2}}{\tau} \nabla \varepsilon \text {. }
$$

Let us now apply the previous considerations in the numerical simulations of laser produced plasma. The plasma expansion is solved in the planar coordinate system in the region above the target surface (Fig. 1). The $y$-axis coincides with the laser beam axis and is directed along the outer normal to the target surface. The plasma evolution is described with the following assumptions: (i) the plasma is in the state of local thermo-dynamical equilibrium and satisfies the quasi-neutrality condition; (ii) the expansion is described in the approximation of a diffusion type equation; (iii) the source term is introduced through the boundary conditions.

In such circumstances, the two-dimensional gas dynamics is described by the equation of concentration

$$
\frac{\partial n}{\partial t}=D \Delta n \text {. }
$$

For the numerical integration, the following initial and boundary conditions are taken:

i) The box integration domain is initially filled with undisturbed gas,

$$
n=n_{0} \quad \text { for } \quad t=0, \quad 0 \leq(x \times y) \leq\left(L_{x} \times L_{y}\right)
$$




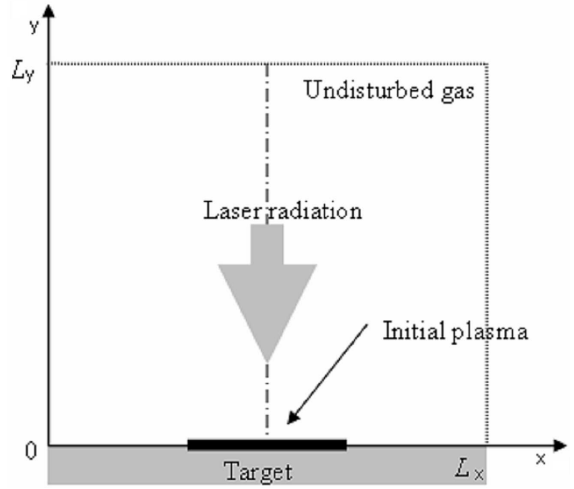

Fig. 1. Integration domain used for the numerical simulation of the laser-produced plasma expansion.
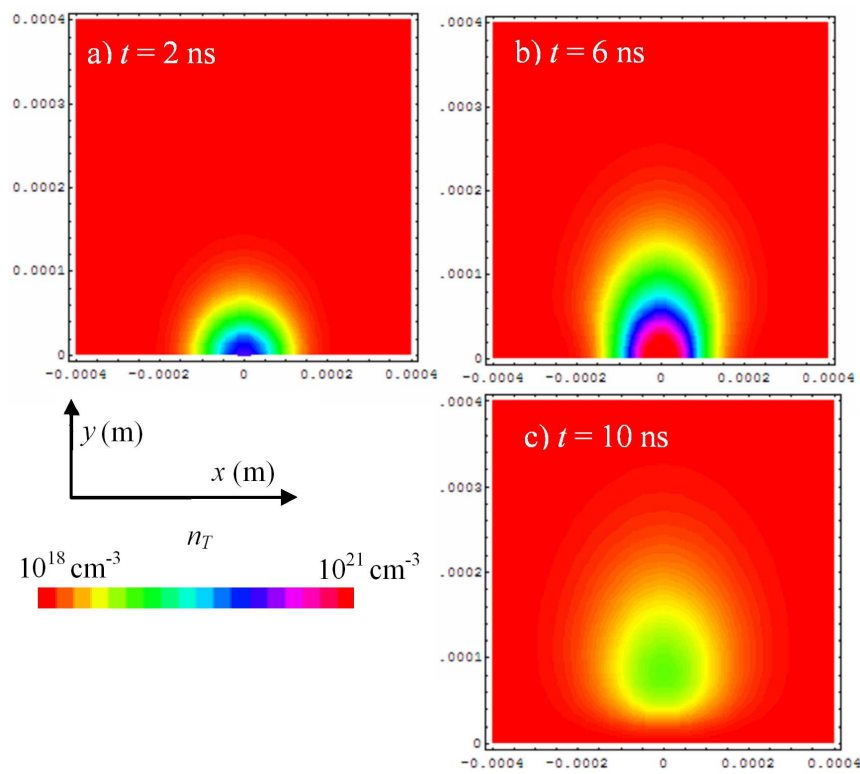

Fig. 2. The contour curves of total atom density for various time moments as resulting from the numerical simulation of the diffusion equation.

that is preserved on the boundaries, $n\left(t, x, L_{y}\right)=$ $n\left(t, L_{x} / 2, y\right)=n\left(t,-L_{x} / 2, y\right)=n_{\max } / 1000$;

ii) The interaction of the laser beam with the target produces a plasma source located on the target surface, which is assumed to have a Gaussian space-time profile

$$
n=n_{\max } \exp \left(-\frac{(t-\tau)^{2}}{\left(\tau_{\mathrm{L}} / 2\right)^{2}}\right) \exp \left(-\frac{x^{2}}{\left(\mathrm{~d}_{\mathrm{L}} / 2\right)^{2}}\right),
$$

with $d_{\mathrm{L}}, \tau_{\mathrm{L}}$ similarly with the laser beam space-time full widths. We underline that the ablation takes place only into a region with a characteristic diameter of about $100 \mu \mathrm{m}$. The maximum atoms density $n_{\max }$ is taken according to the critical electron density $\left(n_{\mathrm{ec}}=3.9 \times\right.$ $10^{21} \mathrm{~cm}^{-3}$ [37]) at the laser wavelength $(\lambda=532 \mathrm{~nm})$.

The diffusion equation of concentration with the initial and boundary conditions is numerically solved us-

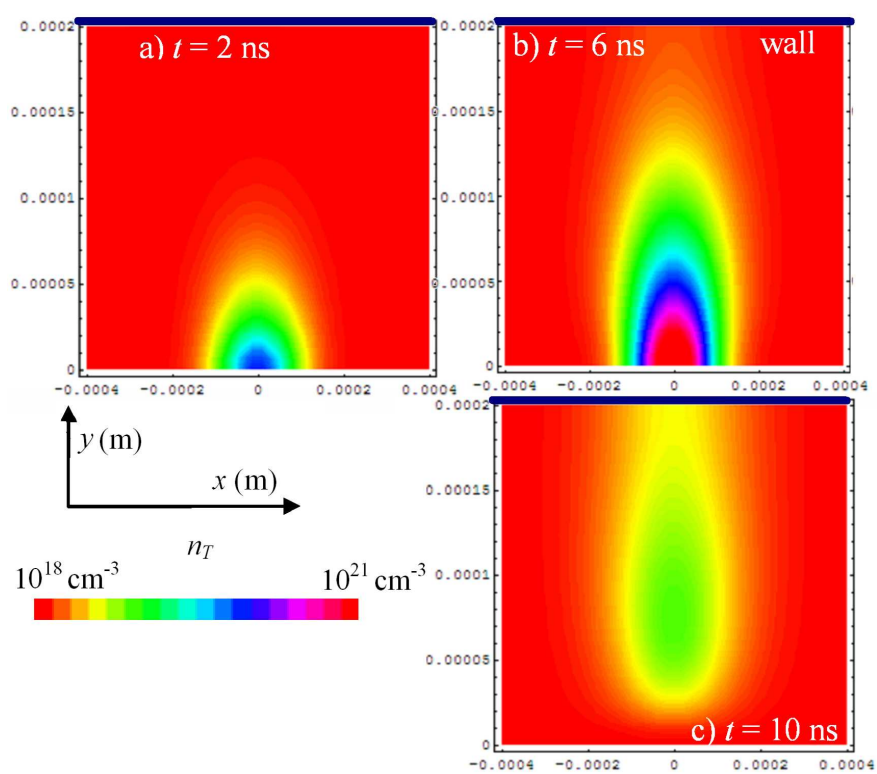

Fig. 3. The contour curves of total atom density for various time moments as resulting from the numerical simulation of the diffusion equation in the presence of a wall.

ing finite differences [38] and the following parameters: $L_{x}=800 \mu \mathrm{m}, L_{y}=400 \mu \mathrm{m}, \tau_{\mathrm{L}}=10 \mathrm{~ns}, d_{\mathrm{L}}=100 \mu \mathrm{m}$, $n_{\max }=1.95 \times 10^{21} \mathrm{~cm}^{-3}, n=n_{\max } / 1000$. Moreover, if the diffusion takes place in the presence of a wall, the previous condition is replaced by $\partial n / \partial y\left(t, x, L_{y}\right)=0$.

In Figs. 2 and 3 the two-dimensional contour curves of the total atom density at the time moments $t=2 \mathrm{~ns}$ (a), $t=6 \mathrm{~ns}(\mathrm{~b})$, and $t=10 \mathrm{~ns}(\mathrm{c})$ are given as obtained from the numerical simulations (Fig. 2 in the absence of wall, Fig. 3 in the presence of wall). The following considerations result: (i) the plasma plume "disappears" by diffusion; (ii) near the wall the plasma plume is "regenerating".

\section{Fractal fluids of convection type behavior}

The inertial principle in its strong covariance form (Nottale's principle $[16,17,27,28]$ ) is reduced to an equation of the Navier-Stokes type (geodesics equation),

$$
\begin{gathered}
\frac{\hat{\mathrm{d}} \mathcal{V}}{\mathrm{d} t}=\frac{\partial \mathcal{V}}{\partial t}+\hat{\mathcal{V}} \cdot \nabla \mathcal{V} \equiv \frac{\partial \mathcal{V}}{\partial t}+\mathcal{V} \cdot \nabla \mathcal{V} \\
-\mathrm{i} \frac{\lambda^{2}}{2 \tau}\left(\frac{\mathrm{d} t}{\tau}\right)^{\left(\frac{2}{D_{\mathrm{F}}}\right)-1} \Delta \mathcal{V}=0
\end{gathered}
$$

with a imaginary viscosity coefficient $\nu$ :

$$
\nu=\mathrm{i} \frac{\lambda^{2}}{2 \tau}\left(\frac{\mathrm{d} t}{\tau}\right)^{\left(\frac{2}{D_{\mathrm{F}}}\right)-1} .
$$

This means that the local complex acceleration field, $\partial \mathcal{V} / \partial t$, the convective term, $\mathcal{V} \cdot \nabla \mathcal{V}$, and the dissipative one, $\Delta \mathcal{V}$, reciprocally compensate in any point of the fractal curve. Moreover, the behavior of the fractal fluid 
is visco-elastic type or hysteretic type. Such results are in agreement with the opinions given in [39, 40]: the fractal fluid can be described by Kelvin-Voight or Maxwell rheological model with imaginary structure coefficient $\nu$.

Two types of motion are distinguished:

i) Rotational motions.

Replacing the complex speed field (13) in geodesics equation (40) and separating the real and the imaginary parts we obtain:

$$
\begin{aligned}
& \frac{\partial \boldsymbol{V}}{\partial t}+\boldsymbol{V} \cdot \nabla \boldsymbol{V}-\boldsymbol{U} \cdot \nabla \boldsymbol{U} \\
& -\frac{\lambda^{2}}{2 \tau}\left(\frac{\mathrm{d} t}{\tau}\right)^{\left(\frac{2}{D_{\mathrm{F}}}\right)-1} \Delta \boldsymbol{U}=0, \\
& \frac{\partial \boldsymbol{U}}{\partial t}+\boldsymbol{U} \cdot \nabla \boldsymbol{V}+\boldsymbol{V} \cdot \nabla \boldsymbol{U} \\
& +\frac{\lambda^{2}}{2 \tau}\left(\frac{\mathrm{d} t}{\tau}\right)^{\left(\frac{2}{D_{\mathrm{F}}}\right)-1} \Delta \boldsymbol{V}=0 .
\end{aligned}
$$

Using the operatorial relations

$$
\begin{gathered}
\nabla(\boldsymbol{V} \cdot \boldsymbol{U})=(\boldsymbol{V} \cdot \nabla) \boldsymbol{U}+(\boldsymbol{U} \cdot \nabla) \boldsymbol{V} \\
+\boldsymbol{V} \times(\nabla \times \boldsymbol{U})+\boldsymbol{U} \times(\nabla \times \boldsymbol{V}), \\
\nabla \boldsymbol{V}^{2}=2(\boldsymbol{V} \cdot \nabla) \boldsymbol{V}+2 \boldsymbol{V} \times(\nabla \times \boldsymbol{V}), \\
\nabla \boldsymbol{U}^{2}=2(\boldsymbol{U} \cdot \nabla) \boldsymbol{U}+2 \boldsymbol{U} \times(\nabla \times \boldsymbol{U}),
\end{gathered}
$$

Eqs. (42a,b) become

$$
\begin{aligned}
& \frac{\partial \boldsymbol{V}}{\partial t}+\nabla\left(\frac{\boldsymbol{V}^{2}}{2}-\frac{\boldsymbol{U}^{2}}{2}\right)-\boldsymbol{V} \times(\nabla \times \boldsymbol{V}) \\
& +\boldsymbol{U} \times(\nabla \times \boldsymbol{U})-\frac{\lambda^{2}}{2 \tau}\left(\frac{\mathrm{d} t}{\tau}\right)^{\left(\frac{2}{D_{\mathrm{F}}}\right)-1} \Delta \boldsymbol{U}=0 \\
& \frac{\partial \boldsymbol{U}}{\partial t}+\nabla(\boldsymbol{V} \cdot \boldsymbol{U})-\boldsymbol{V} \times(\nabla \times \boldsymbol{U})-\boldsymbol{U} \times(\nabla \times \boldsymbol{V}) \\
& +\frac{\lambda^{2}}{2 \tau}\left(\frac{\mathrm{d} t}{\tau}\right)^{\left(\frac{2}{D_{\mathrm{F}}}\right)-1} \Delta \boldsymbol{V}=0
\end{aligned}
$$

and moreover, introducing "the vortices"

$$
\begin{aligned}
& \boldsymbol{\Omega}_{V}=\frac{1}{2}(\nabla \times \boldsymbol{V}), \quad \boldsymbol{\Omega}_{U}=\frac{1}{2}(\nabla \times \boldsymbol{U}) \\
& \frac{\partial \boldsymbol{V}}{\partial t}+\nabla\left(\frac{\boldsymbol{V}^{2}}{2}-\frac{\boldsymbol{U}^{2}}{2}\right)-2 \boldsymbol{V} \times \boldsymbol{\Omega}_{V}+2 \boldsymbol{U} \times \boldsymbol{\Omega}_{U} \\
& -\frac{\lambda^{2}}{2 \tau}\left(\frac{\mathrm{d} t}{\tau}\right)^{\left(2 / D_{\mathrm{F}}\right)-1} \Delta \boldsymbol{U}=0 \\
& \frac{\partial \boldsymbol{U}}{\partial t}+\nabla(\boldsymbol{V} \cdot \boldsymbol{U})-2 \boldsymbol{V} \times \boldsymbol{\Omega}_{U}-2 \boldsymbol{U} \times \boldsymbol{\Omega}_{V} \\
& +\frac{\lambda^{2}}{2 \tau}\left(\frac{\mathrm{d} t}{\tau}\right)^{\left(2 / D_{\mathrm{F}}\right)-1} \Delta \boldsymbol{V}=0
\end{aligned}
$$

Relations (46a,b) characterize the transport of the specific momentum both at the differentiable scale, (46a), and the non-differentiable scale (46b). It is conditioned by the inertial effects, $\left(\nabla \boldsymbol{V}^{2}, \nabla \boldsymbol{U}^{2}\right),(\nabla(\boldsymbol{V} \cdot \boldsymbol{U}))$, rotational effects $\left(\boldsymbol{V} \times \boldsymbol{\Omega}_{V}, \boldsymbol{U} \times \boldsymbol{\Omega}_{U}\right),\left(\boldsymbol{V} \times \boldsymbol{\Omega}_{U}, \boldsymbol{U} \times \boldsymbol{\Omega}_{V}\right)$, and dissipative effects $((\Delta \boldsymbol{V}, \Delta U))$;

ii) Irrotational motions.

In this case $\nabla \times \mathcal{V}=0$

so that the speed field (13) can be expressed through the gradient of a scalar function $\Phi$,

$$
\mathcal{V}=\nabla \Phi
$$

named the scalar potential of the complex speed field, $\Phi=\operatorname{Re} \Phi+\mathrm{i} \operatorname{Im} \Phi$.

Substituting Eq. (48) in Eq. (40) and using the operatorial relationship

$$
\frac{\partial}{\partial t} \nabla=\nabla \frac{\partial}{\partial t}
$$

it results

$$
\begin{aligned}
& \nabla\left[\frac{\partial \Phi}{\partial t}+\frac{1}{2}(\nabla \Phi)^{2}-\mathrm{i} \frac{\lambda^{2}}{\tau}\left(\frac{\mathrm{d} t}{\tau}\right)^{\left(\frac{2}{D_{\mathrm{F}}}\right)-1} \Delta \Phi\right] \\
& =0
\end{aligned}
$$

and by integration, a Bernoulli type equation

$$
\frac{\partial \Phi}{\partial t}+\frac{1}{2}(\nabla \Phi)^{2}-\mathrm{i} \frac{\lambda^{2}}{2 \tau}\left(\frac{\mathrm{d} t}{\tau}\right)^{\left(\frac{2}{D_{\mathrm{F}}}\right)-1} \Delta \Phi=F(t)
$$

with $F(t)$ a function which depends only on time. Particularly, for $\Phi$ of the form $[27,28]$

$$
\Phi=-\mathrm{i} \frac{\lambda^{2}}{\tau}\left(\frac{\mathrm{d} t}{\tau}\right)^{\left(\frac{2}{D_{\mathrm{F}}}\right)-1} \ln \psi,
$$

where $\psi$ is a new complex scalar function, Eq. (51) with the operatorial identity

$$
\frac{\Delta \psi}{\psi}=\Delta \ln \psi+(\nabla \ln \psi)^{2}
$$

takes the form

$$
\begin{aligned}
& \frac{\lambda^{4}}{4 \tau^{2}}\left(\frac{\mathrm{d} t}{\tau}\right)^{\left(\frac{4}{D_{\mathrm{F}}}\right)-2} \Delta \psi+\mathrm{i} \frac{\lambda^{2}}{2 \tau}\left(\frac{\mathrm{d} t}{\tau}\right)^{\left(\frac{2}{D_{\mathrm{F}}}\right)-1} \frac{\partial \psi}{\partial t} \\
& \quad+\frac{F(t)}{2} \psi=0 .
\end{aligned}
$$

From here, "Schrödinger" type geodesics result for $F(t) \equiv$ 0 , i.e.

$$
\begin{aligned}
& \frac{\lambda^{4}}{4 \tau^{2}}\left(\frac{\mathrm{d} t}{\tau}\right)^{\left(\frac{4}{D_{\mathrm{F}}}\right)-2} \Delta \psi+\mathrm{i} \frac{\lambda^{2}}{2 \tau}\left(\frac{\mathrm{d} t}{\tau}\right)^{\left(\frac{2}{D_{\mathrm{F}}}\right)-1} \frac{\partial \psi}{\partial t} \\
& \quad=0
\end{aligned}
$$

— for more details see Sect. 4.3 from [28]. Particularly, for the movement on fractal curves of the Peano type, i.e. in the fractal dimension $D_{\mathrm{F}}=2$, and Compton's length and temporal scales,

$$
\lambda=\frac{\hbar}{2 m_{0} c}, \quad \tau=\frac{\hbar}{m_{0} c^{2}},
$$

Eq. (55) takes the Schrödinger standard form

$$
\frac{\hbar^{2}}{2 m} \Delta \psi+\mathrm{i} \hbar \frac{\partial \psi}{\partial t}=0
$$

Also, a fractal hydrodynamic model can be developed. Thus, by replacing the complex speed field (48) in Eq. (40), and separating the real and imaginary parts we obtain 


$$
\begin{aligned}
& m_{0} \frac{\partial \boldsymbol{V}}{\partial t}+m_{0} \boldsymbol{V} \cdot \nabla \boldsymbol{V}=-\nabla(Q), \\
& \frac{\partial \boldsymbol{U}}{\partial t}+\nabla(\boldsymbol{V} \cdot \boldsymbol{U})+\frac{\lambda^{2}}{2 \tau}\left(\frac{\mathrm{d} t}{\tau}\right)^{\left(\frac{2}{D_{\mathrm{F}}}\right)-1} \Delta \boldsymbol{V}=0,
\end{aligned}
$$

where $Q$ is the fractal potential

$$
Q=-\frac{m_{0} \boldsymbol{U}^{2}}{2}-m_{0} \frac{\lambda^{2}}{2 \tau}\left(\frac{\mathrm{d} t}{\tau}\right)^{\left(\frac{2}{D_{\mathrm{F}}}\right)-1} \nabla \cdot \boldsymbol{U} .
$$

The explicit form of the complex speed field is given by means of the expression

$$
\psi=\sqrt{\rho} \mathrm{e}^{\mathrm{i} S}
$$

with $\rho$ the amplitude and $S$ the phase. Then Eq. (48) with

$$
\Phi=-\mathrm{i} \frac{\lambda^{2}}{\tau}\left(\frac{\mathrm{d} t}{\tau}\right)^{\left(\frac{2}{D_{\mathrm{F}}}\right)-1} \ln \left(\sqrt{\rho} \mathrm{e}^{\mathrm{i} S}\right)
$$

involves the complex velocity field components

$$
\begin{aligned}
& \boldsymbol{V}=\frac{\lambda^{2}}{\tau}\left(\frac{\mathrm{d} t}{\tau}\right)^{\left(\frac{2}{D_{\mathrm{F}}}\right)-1} \nabla S, \\
& \boldsymbol{U}=\frac{\lambda^{2}}{2 \tau}\left(\frac{\mathrm{d} t}{\tau}\right)^{\left(\frac{2}{D_{\mathrm{F}}}\right)-1} \nabla \ln \rho,
\end{aligned}
$$

while the fractal potential (59) is given by the simple expression

$$
Q=-m_{0}\left(\frac{\mathrm{d} t}{\tau}\right)^{\left(\frac{2}{D_{\mathrm{F}}}\right)-1} \frac{\Delta \sqrt{\rho}}{\sqrt{\rho}}
$$

- for other details see [19].

With Eqs. (62a,b), Eq. (58b) takes the form

$$
\nabla\left(\frac{\partial \ln \rho}{\partial t}+\boldsymbol{V} \cdot \nabla \ln \rho+\nabla \cdot \boldsymbol{V}\right)=0
$$

or, by integration with $\rho \neq 0$ :

$$
\frac{\partial \rho}{\partial t}+\nabla \cdot(\rho \boldsymbol{V})=T(t)
$$

with $T(t)$, a function which depends only on time.

Equation (58a) corresponds to the momentum conservation law, while Eq. (65), with $T(t) \equiv 0$, to the probability density conservation law. So equations

$$
\begin{aligned}
& m_{0}\left(\frac{\partial \boldsymbol{V}}{\partial t}+\boldsymbol{V} \cdot \nabla \boldsymbol{V}\right)=-\nabla(Q), \\
& \frac{\partial \rho}{\partial t}+\nabla \cdot(\rho \boldsymbol{V})=0
\end{aligned}
$$

with $Q$ given by (63), form the fractal hydrodynamic equations in the fractal dimension $D_{\mathrm{F}}$. The fractal potential (63) is induced by the non-differentiable space-time (for more details see [19]).

\section{Conclusions}

The main conclusions of the present paper are the following:

i) A short reminder of the Nottale scale relativity theory in correspondence with Cresson's mathematical procedures is given; ii) The synchronization of the movements at different scales gives conductive properties to the fractal fluid. In such a context, by numerical simulation of plasma expansion, it results that plasma plume disappears by diffusion or is self-generating near a wall;

ii) The non-synchronous movement at different scales gives conductive properties to the fractal fluid. In such a context, at Compton scale, the irrotational fractal fluid is described by the Schrödinger equation.

\section{Acknowledgments}

The authors wish to thank the referees for their pertinent observations. Part of the present work was supported by IDEI_605 contract of CNCSIS.

\section{References}

[1] L. Nottale, Int. J. Mod. Phys. A 7, 4899 (1992).

[2] L. Nottale, Fractal Space-Time and Microphysics: Towards a Theory of Scale Relativity, World Scientific, Singapore 1993.

[3] L. Nottale, Astron. Astrophys. 327, 867 (1997).

[4] L. Nottale, Chaos, Solitons Fractals 9, 1051 (1998).

[5] L. Nottale, Chaos, Solitons Fractals 10, 459 (1999).

[6] L. Nottale, Chaos, Solitons Fractals 16, 539 (2003).

[7] D. Da Rocha, L. Nottale, Chaos, Solitons Fractals 16 , 565 (2003).

[8] L. Nottale, Chaos, Solitons Fractals 25, 797 (2005).

[9] G.N. Ord, J. Phys. A 16, 1869 (1983).

[10] Quantum Mechanics, Diffusion and Chaotic Fractals, Eds. M.S. El Naschie, O.E. Rösler, I. Prigogine, Elsevier, Oxford 1995.

[11] M.S. El Naschie, Chaos, Soliton Fractals 22, 495 (2004).

[12] M.S. El Naschie, Chaos, Soliton Fractals 14, 649 (2002).

[13] M.S. El Naschie, Chaos, Soliton Fractals 12, 1167 (2002).

[14] M.S. El Naschie, Chaos, Soliton Fractals 25, 969 (2005).

[15] M.S. El Naschie, Chaos, Soliton Fractals 27, 9 (2006).

[16] M.N. Célérier, L. Nottale, J. Phys. A, Math. Gen. 37, 931 (2004).

[17] L. Nottale, M.N. Célérier, arXiv:0711.2418v1 (2009).

[18] L. Nottale, arXiv:0901.1270 (2009).

[19] L. Nottale, J. Phys. A, Math. Theor. 42, 275306 (2009).

[20] B.B. Mandelbrot, Fractals: Form, Chance and Dimension, W.H. Freeman, San Francisco 1977.

[21] B.B. Mandelbrot, The Fractal Geometry of Nature, W.H. Freeman, San Francisco 1983.

[22] B.B. Mandelbrot, Fractal and Scaling in Finance, Springer-Verlag, Berlin 1997. 
[23] I. Gottlieb, M. Agop, G. Ciobanu, A. Stroe, Chaos, Solitons Fractals 30, 380 (2006).

[24] P. Mora, Phys. Rev. Lett. 90, 185002 (2003).

[25] M. Agop, A. Harabagiu, P. Nica, Acta Phys. Pol. A 113, 1557 (2008).

[26] M. Agop, P. Nica, P.D. Ioannou, O. Malandraki, I. Gavanas-Pahomi, Chaos, Solitons Fractals 34, 1704 (2007).

[27] L. Nottale, Chaos, Solitons Fractals 6, 399 (1995).

[28] L. Nottale, Chaos, Solitons Fractals 7, 877 (1996).

[29] J. Cresson, Int. J. Geometric Meth. Mod. Phys. 3, 1395 (2006)

[30] J. Cresson, J. Math. Anal. Appl. 307, 48 (2005).

[31] J. Cresson, F. Ben Adda, Chaos, Solitons Fractals 19, 1323 (2004).

[32] J. Cresson, J. Math. Phys. 44, 4907 (2003).
[33] J. Cresson, Chaos, Solitons Fractals 14, 553 (2002).

[34] J. Cresson, F. Ben Adda, J. Math. Anal. Appl. 262, 721 (2001).

[35] J. Cresson, J.-N. Denarie, in: Noise, Oscillators and Algebraic Randomness Ed. M. Planat, Springer-Verlag, Berlin 2000, p. 305.

[36] J. Cresson, F. Ben Adda, C.R. Acad. Sci. Paris 330, 261 (2000).

[37] L. Spitzer, Physics of Fully Ionized Gases, Interscience, New York 1962.

[38] O.C. Zienkiewicz, R.L. Taylor, The Finite Element Method, McGraw-Hill, New York 1991.

[39] Y. Imry, Introduction to Mesoscopic Physics, Oxford University Press, Oxford 2002.

[40] D.K. Ferry, S.M. Goodnick, Transport in Nanostructures, Cambridge University Press, Cambridge 1997. 